\title{
Aerodynamic Investigation about the Cause of Check-mark Stain on the Galvanized Steel Surface
}

\author{
Hyun Gi YOON, ${ }^{1)}$ Gi Jang $\mathrm{AHN}_{,}{ }^{2)}$ Sang Jun $\mathrm{KIM}^{3)}$ and Myung Kyoon CHUNG ${ }^{11}$ \\ 1) Korea Advanced Institute of Science and Technology (KAIST), 4120, Yuseong-gu, Deajeon, 305-701, Republic of Korea. \\ E-mail: yoonhyungi@gmail.com $\quad$ 2) Samsung SDI, Gongsedong, Giheung-gu, Yongin-si, Gyeonggi-do, South Korea. \\ 3) Graduate Institute of Ferrous Technology (GIFT), POSTECH, Pohang, South Korea.
}

(Received on March 30, 2009; accepted on August 11, 2009)

\begin{abstract}
When galvanized steel strip is produced through a continuous hot-dip galvanizing process, the thickness of the adhered zinc film is controlled by impinging a thin plane nitrogen gas jet. In such a gas wiping process frequently there appears stain of check-mark shape. The check-mark stain is caused by non-uniform zinc coating over the surface. In order to find the main reason of the appearance of the check-mark stain, the unsteady compressible flow field is numerically simulated by using LES turbulence model for two cases of $L / d=6.67$ in the Case 1, 11.54 in the Case 2 where $d$ is the nozzle width and $L$ is the lip-to-plate distance. It was found that there are alternating plane-wise vortices near the impinging stagnation region, and that such alternating vortices move almost periodically to the right and to the left sides on the stagnation line due to the jet flow instability and alternating pressure peaks along the stagnation line. Since higher stagnation pressure removes more molten zinc adhered on the surface, the zinc coating thickness is thinner there. In addition, since the strip moves upward at a constant speed, the non-uniform coating surface is formed with a variety of patterns like " $\mathrm{W}$ ", " $\mathrm{V}$ " and " $\mathrm{X}$ ". The angle of the check-mark was calculated by using both the moving speeds of the steel strip and the sidewise movement of the vortices along the stagnation line. It was favorably compared with the experimental measurement.
\end{abstract}

KEY WORDS: Slot impinging jet; coherent structure; vortex pair; LES; galvanized steel.

\section{Introduction}

Since the galvanized steel strip has good corrosion resistance and good characteristics for painting, welding and manufacturing, it is widely used in a variety of industrial fields such as civil engineering, automobile, shipbuilding and home appliance industries. It is produced by a continuous hot dip galvanizing process. A heat-treated steel strip is passed through a molten zinc bath and is drawn up with the molten zinc adhered on both strip surfaces as shown in Fig. 1. Usually the thickness of the adhered zinc film is about 10 times thicker than desired thickness. In order to remove the excessive zinc, the steel strip is vertically drawn up between a pair of two-dimensional opposing plane gas jets. In this way the unnecessary molten zinc is wiped down by the impinging gas jets. This wiping mechanism has been well investigated theoretically and experimentally. ${ }^{1-4)}$ The nozzle of the nitrogen gas jet is called the air knife system and this manufacturing method is called the gas wiping process. This method which was put to practical use in the midtwenty century is widely used in most of the commercial plants because of good productivity and easy control of the zinc coating thickness. However, such a gas wiping process has two technical problems concerning the uniformity of the coated film thickness. One is the edge over coating (EOC) problem in both the strip edge regions and the other is the check-mark stain problem in the interior region. ${ }^{5-7)}$
The edge over coating problem is that the thickness of the zinc film near the strip edge regions is $1.4-1.8$ times thicker than that on the surface of the interior strip region. The principal cause of the EOC was found by Kim et al. ${ }^{7)}$ They simulated numerically the turbulent flow field around the gas wiping region formed by the two impinging/opposing jets and revealed that due to the oscillation of the opposing jets in the outside region of the strip edge, the static pressure decreases significantly toward the strip edge, which dramatically reduces the zinc wiping force. After this research, Ahn et al. ${ }^{8)}$ suggested a feasible solution for EOC

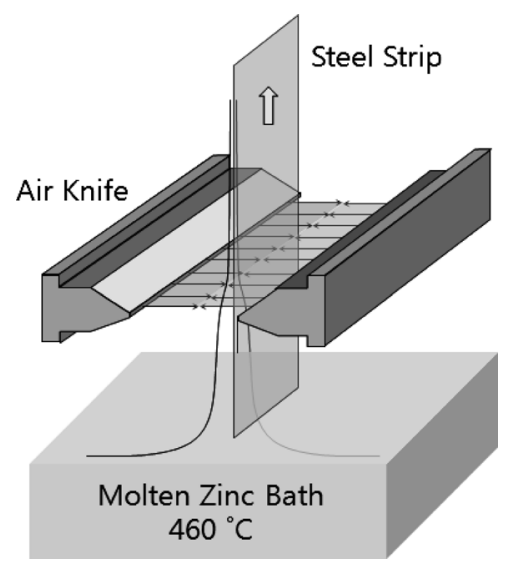

Fig. 1. Schematic diagram of a hot-dip galvanizing process. 
by using the Coanda flow effect to remove the oscillation of the opposing jets outside of the strip edge.

The check-mark stain is the oblique patterns that appear on the coated film surface after the gas wiping process. Depending on its seriousness, the check-mark stain is usually classified into four grades as shown in Fig. 2. Each arrow indicates the moving direction of steel strip. The first grade has no problem on the surface. The surface pattern of the 2nd grade check-mark stain has surface defects such as irregular points or lines. On the other hand, the 3rd-4th grade check-mark stains have surface defects looking like oblique patterns such as "W", "V" or "X". The check-mark stain causes many problems such as irregularity in the electrical and thermal characteristics and the diffused reflection on the coated surface. When such defects are serious, the steel strip can not be used in manufacturing the end products. So, it is the main reason to lower profitability and productivity of the zinc-coated still strip. Even though the checkmark defect is one of the chronic problems deteriorating the quality, its main mechanism is not fully understood yet. From the results of many experiments and numerical simulations about the 2-dimensional impinging jet, it can also be seen that the plane impinging jet is unsteady flow and its symmetry is collapsed by the repeated formation and destruction of vortices in the upper and lower part of the plane jet at high Reynolds number. ${ }^{9-15)}$

The objective of the present study is to clarify the cause of the check-mark stain by scrutinizing in depth the numerical data obtained from numerical simulations of the unsteady compressible flow field which is formed when twodimensional plane gas jets collide with a moving vertical strip in a close distance. Studying the unsteady flow field obtained from numerical simulation results of our preceding researches, it was found that the flow field near the plane impinging jet ejected from the air-knife to the steel plate is not steady state but unsteady, and that large turbulent eddies change with time in a regular pattern. It was also observed that the instantaneous surface stagnation pressure varies in almost sinusoidal pattern along the impingement line on the strip surface and the points of peak pressure move right or left alternatively at a nearly constant rate. At the point of peak pressure, the adhered molten zinc is wiped at a maximum rate and thus the coating thickness becomes thinnest there. Combination of this alternating sidewise movement of the peak pressure points with the upward moving steel strip at a constant speed is thought to result in the check-mark stain on the coated strip surface. Figure 3 is drawn to illustrate such a check-mark formation process.

In order to understand the phenomenon of the checkmark stain based on the fluid dynamic point of view, the 3D unsteady compressible flow is studied numerically by using the LES turbulence model. Smagorinsky-Lilly SGS model is used in the present LES study. ${ }^{16}$

\section{Numerical Analysis}

Since the Mach number of the plane impinging jet is around $0.3-0.8$ in the gas wiping process, the unsteady $3-\mathrm{D}$ compressible turbulent flow is numerically simulated by using the commercial code, FLUENT to observe the char-

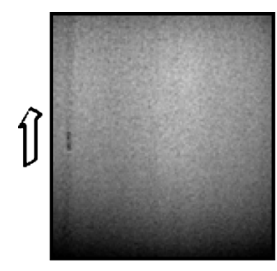

(a) $1^{\text {st }}$ grade

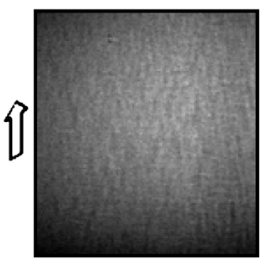

(c) $3^{\text {rd }}$ grade

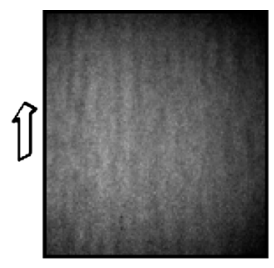

(b) $2^{\text {nd }}$ grade

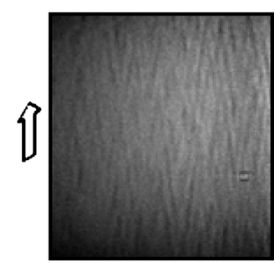

(d) $4^{\text {th }}$ grade
Fig. 2. Photograph of check-mark with grade, (a) 1st grade, (b) 2nd grade, (c) 3rd grade, (d) 4th grade.

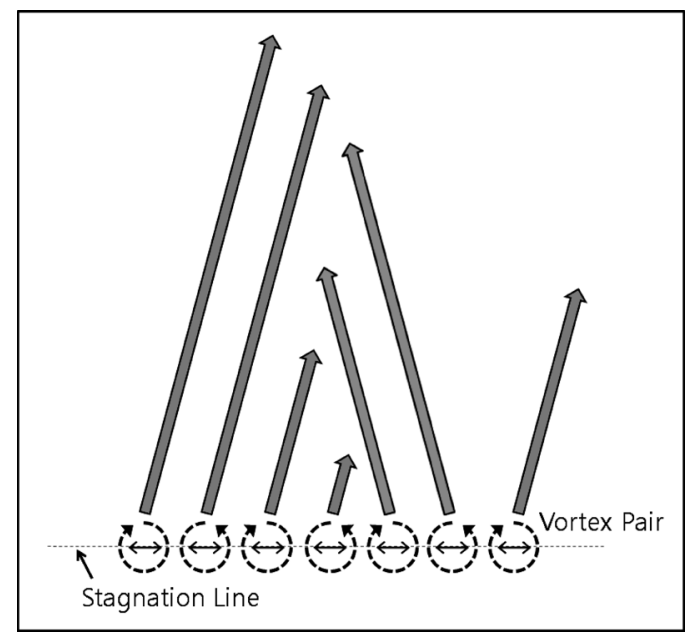

Fig. 3. Schematic diagram of an alternating vortex pairs on the stagnation line.

acteristics of the flow filed. Boundary conditions and the computational domain of the gas wiping system are shown in Fig. 4. The length in the $z$-direction is $200 \mathrm{~mm}$ which is one-fifth of the steel strip width in the zinc coating plant. The opposing jet region in the outside of the strip edge is not considered because the check-mark stain appears mostly in the interior region. It is assumed that the flow field is homogeneous in the span wise direction, and so the symmetric condition is given at the both sides of the calculation domain. The steel strip moves from bottom to top, and the calculation domain in the vertical direction is $70 \mathrm{~mm}$. The atmospheric pressure condition is used at the top and bottom boundaries. The symbols $d$ and $L$ in Fig. 4 represent the lip width of the air knife and the distance from the exit of the air knife to the steel strip, respectively. The coordinate origin is displayed in Fig. 4. At this time, $x$, $y$-coordinate origins are taken at the wall of the steel strip and the lower end of the exit of the air knife, respectively. The same coordinate origin is used in all numerical cases.

The inside stagnation pressure $\left(P_{0}\right)$ and temperature $\left(T_{0}\right)$ of the air-knife are given with the respective values under which the check-mark stain appears in the gas wiping 


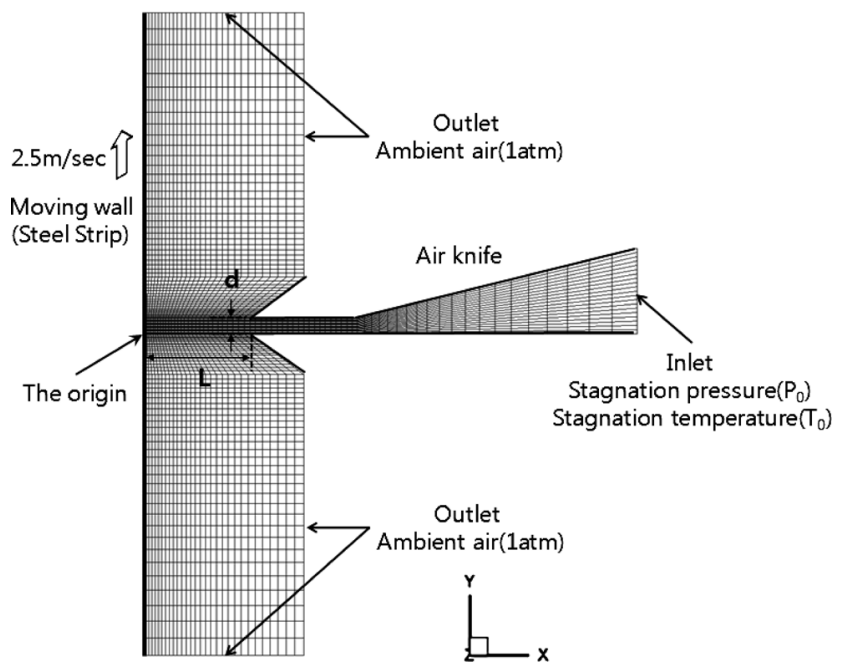

Fig. 4. Calculation domain and boundary condition of the air knife system.

Table 1. Geometry and calculation conditions.

\begin{tabular}{|c|c|c|c|c|c|c|}
\hline Case & $\mathrm{d}$ & $\mathrm{L}$ & $\mathrm{L} / \mathrm{d}$ & $\mathrm{P}_{0}$ & $\mathrm{~T}_{0}$ & Grade of check mark \\
\hline 1 & $1.5 \mathrm{~mm}$ & $10 \mathrm{~mm}$ & 6.67 & $25 \mathrm{kPa}$ & $340 \mathrm{~K}$ & $3^{\text {rd }}$ grade \\
\hline 2 & $1.3 \mathrm{~mm}$ & $15 \mathrm{~mm}$ & 11.54 & $25 \mathrm{kPa}$ & $340 \mathrm{~K}$ & Weak $2^{\text {nd }}$ grade \\
\hline
\end{tabular}

process by the actual air knife. Working fluid is the nitrogen gas used also in the actual process. The grade of the checkmark, geometrical shape of the air knife and inside operating stagnation conditions are described at Table 1. The surface of the steel strip is treated as a wall moving at a constant speed of $2.5 \mathrm{~m} / \mathrm{s}$ in positive $y$-direction at $x=0$. Large Eddy Simulation (LES) technique is used to produce the turbulent flow date of the impinging jet. Time step of the unsteady solver was taken to be $\Delta t=1.0 \times 10^{-4} \mathrm{~s}$, which is about $1 / 30$ of the pressure fluctuation period on the impingement stagnation line on the strip surface. The smallest grid size is taken to be $0.1 \mathrm{~mm}$ in the jet-plate impinging region. In the $z$-direction, the grids are uniformly distributed, whereas in the $y$-direction, the first grid spacing is $0.1 \mathrm{~mm}$ near the impinging region and it is increased at a successive rate in the $\pm y$-direction. Using this grid spacing strategy, about a total of three millions structured grids were formed with finer meshes in the impinging region as shown in Fig. 4. The averaged Courant number of this grid system is about 2 under these calculation conditions. ${ }^{16)}$ The PISO algorism was used as a pressure-velocity coupling, and as a discretization method, bounded central differencing method was applied, which is appropriate for LES technique. ${ }^{16)}$

\section{Description of the 3-D Unsteady Compressible Flow Field in the Gas Wiping Process}

Simulation result for the 3rd grade check-mark stain of Case 1 in Table 1 will be explained in the first place. Figure 5 shows the velocity contour of the nitrogen gas flow on the $x y$-plane at the middle of the $z$-direction $(z=100 \mathrm{~mm})$. The maximum jet velocity obtained by the simulation is about $230 \mathrm{~m} / \mathrm{s}$. It is favorably compared with $232 \mathrm{~m} / \mathrm{s}$ that is obtained by using the compressible flow equation for the exit velocity from a reservoir under the prescribed stagnation pressure and temperature. ${ }^{17)}$ In general, if a gas jet collides

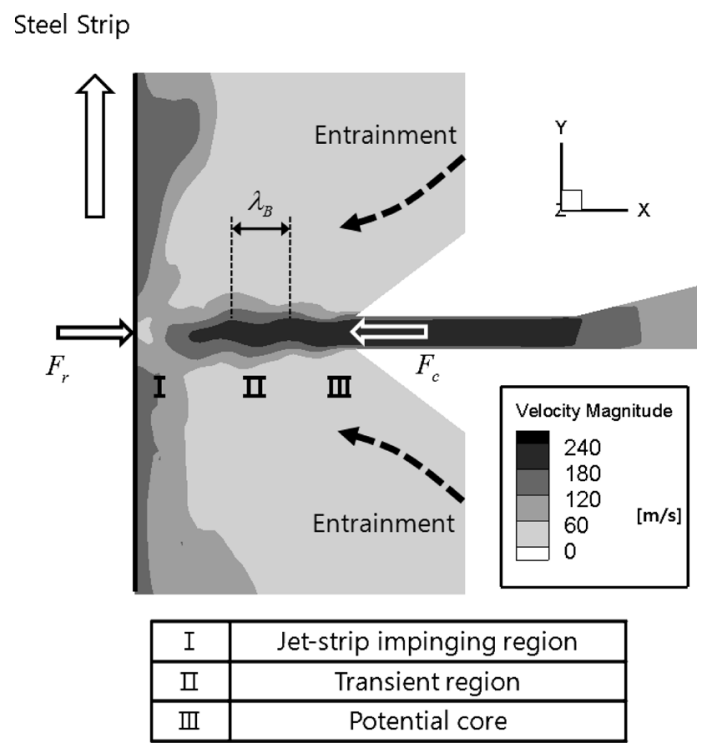

Fig. 5. Velocity magnitude contours, buckling wavelength and flow regions of the impinging jet.

normally on a moving strip, the gas jet is bent toward the moving direction of the strip. ${ }^{18,19)}$ It is known that faster the jet velocity relative to the strip movement, smaller the bending of the gas jet near the impingement. In the present study, since the gas jet velocity is very large in comparison with the moving strip speed, bending of the gas jet is not observed in Fig. 5.

The impinging gas jet field can be divided into three regions; jet-strip impinging region (I), transient region (II) and potential core (III) as shown in Fig. 5. The shape of the potential core is an isosceles triangle with about $3.5 \mathrm{~d}$ of its height, and the width of the potential core gets gradually thinner with the downstream distance from the jet exit in the general free shear jet. But, the length of the potential core is extended in the present air knife system since the impingement distance is very short and extensive entrainment of the surrounding air takes place in proportion to the high velocity of the jet.

Furthermore, the buckling is observed at the center of the potential core in Fig. 5. It is known that the buckling of the jet center plane is one of the potential flow phenomena; it is not due to viscous or turbulent effect. ${ }^{20)}$ The buckling wavelength, $\lambda_{B}$, given by the following equation. ${ }^{20)}$

$$
\lambda_{B}=2 \pi \sqrt{I / A}
$$

Here $I$ is the area moment of inertia and $A$ is the crosssectional area. For a 2-dimensional jet with width of $d$, the buckling wavelength becomes $\lambda_{B} / d=\pi / \sqrt{3}$. Since $d=1.5$ $\mathrm{mm}$ for our Case 1, we obtain the buckling wavelength of about $2.72 \mathrm{~mm}$. Numerical result depicted in Fig. 5 shows that the mean length of the buckling wavelength is about $3 \mathrm{~mm} \pm 20 \%$. The numerical result is reasonably consistent with the analytic computation.

Figures 6(a) and 6(b) show the normalized velocity vectors and static pressure (gauge) contours of the nitrogen gas flow on the $x y$-plane at the middle of the $z$-direction $(z=100 \mathrm{~mm})$, respectively. Entrainment of the air around the air knife into the jet can be observed by the velocity 


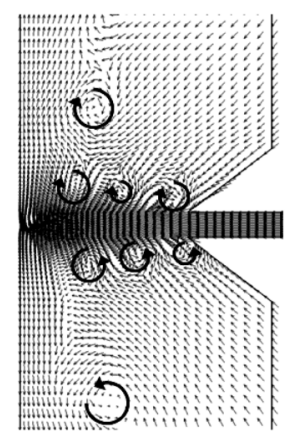

(a) Normalized velocity vectors

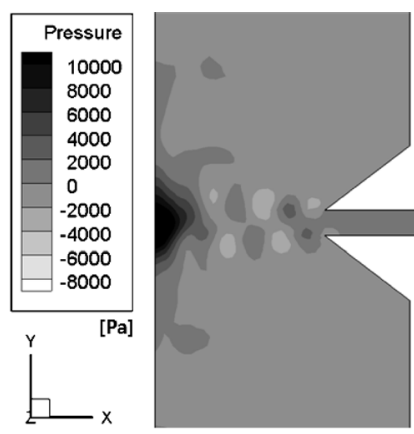

(b) Static pressure contours
Fig. 6. Vortex pairs in the upper and lower part of the jet and static pressure contours. (a) Normalized velocity vectors, (b) static pressure contours.

vectors. And opposed vortices are observed above and below the gas jet center plane. ${ }^{21,22)}$ These vortices move upward or downward direction along the strip after the collision and they grow continuously. Compared with the pressure field depicted in the right hand side in Fig. 6, it is confirmed that the center of the vortex has a relatively low pressure as compared with the outer region. In this manner, non-uniform and unstable flow filed is formed near the transient and the jet-strip impinging region. ${ }^{23-25)}$

Jet impingement region can be further divided into two regions; inner and outer impinging regions. Inner impinging region is where the gas jet collides on the strip surface nearly normally. Outer impinging region is where the outer part of the jet that diffused slightly in $y$-direction collides on the strip surface obliquely.

In the inner impinging region, the velocity vectors have all of 3-dimensional components; sidewise component in $z$ direction, normal component in $x$-direction and stream-wise component in $y$-direction. Figure 7 shows the static pressure contours on the $x z$-plane at $y=1.4 \mathrm{~mm}$. The static pressure varies almost periodically between $5 \mathrm{kPa}$ and $18 \mathrm{kPa}$ along the impingement stagnation line on the surface of the steel strip. Because of this sinusoidal distribution of surface pressure, the gas moves sidewise alternatively in the inner impinging region. In addition it can be observed in Fig. 7 that the static pressure is distributed in a wavelike manner at the far distance from the strip, which confirms the appearance of buckling of the jet center line.

In order to closely investigate the flow characteristics of the nitrogen gas in the inner impinging region, $z$-velocity vector components, static pressure contours and $y$-vorticity contours are displayed on the $y z$-plane at $x=0.1 \mathrm{~mm}$ in Fig. 8. The dash-dotted lines indicate the stagnation lines. The region shown in dotted line in Fig. 8(a) shows the impingement pressure region. Refer to the explanation at Fig. 12. It can be seen that the sidewise velocity component changes its sign and the magnitude varies periodically along the stagnation line in Fig. 8(a). And, high and low static pressure is formed repeatedly along the stagnation line as shown in Fig. 8(b). On comparing Fig. 8(a) with Fig. 8(b), it is found that the static pressure has the minimum value at the point where the sidewise velocity has the maximum value. Meanwhile for $y$-vorticity contours in Fig. 8(c), a positive vorticity means the clockwise rotation on $y$-axis and a negative value means the counterclockwise rotation.

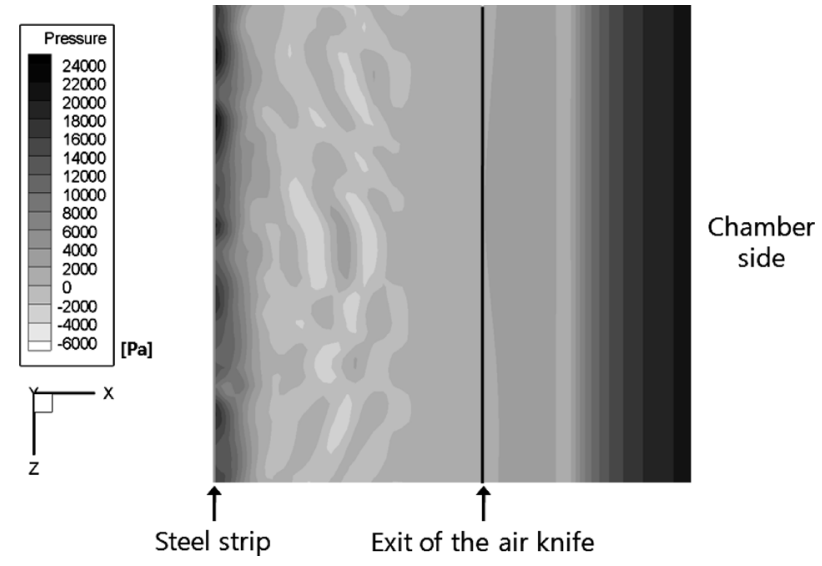

Fig. 7. Static pressure contours on $x z$-plane at $y=1.4 \mathrm{~mm}$.

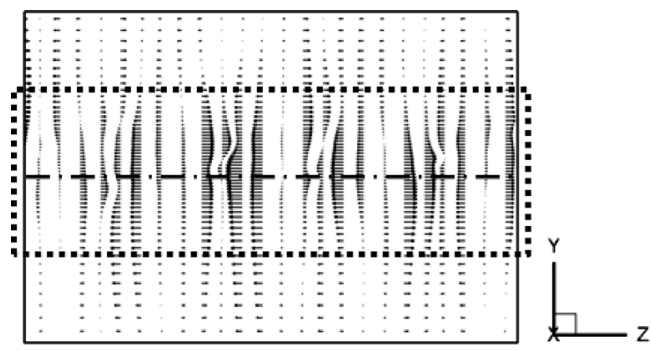

(a) z-velocity vector components

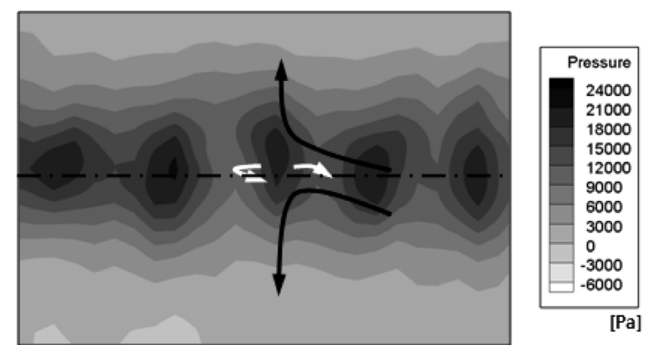

(b) Static pressure contours

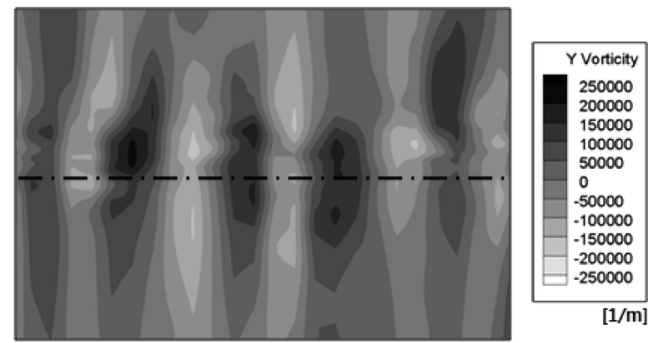

(c) Y-vorticity contours

Fig. 8. Relationship between velocity components, static pressure and $y$-vorticity in the stagnation line. (a) $Z$-velocity vector components, (b) static pressure contours, (c) $Y$ vorticity contours.

In the figure, a negative and positive sign of the vorticity is appeared alternatively along the stagnation line. This implies that the nitrogen gas locally approaches or goes away from the steel strip in the vertical direction. In other words local non-zero $y$-vorticity component generated near the steel strip gives rise to the vertical velocity component in the impinging jet. This phenomenon is the local 3-dimensional flow characteristics obtained by using the LES technique. Such detailed flow field can not be generated by 2-D or 3-D RANS equations employing $k-\varepsilon$ or $k-\omega$ turbulence model. 


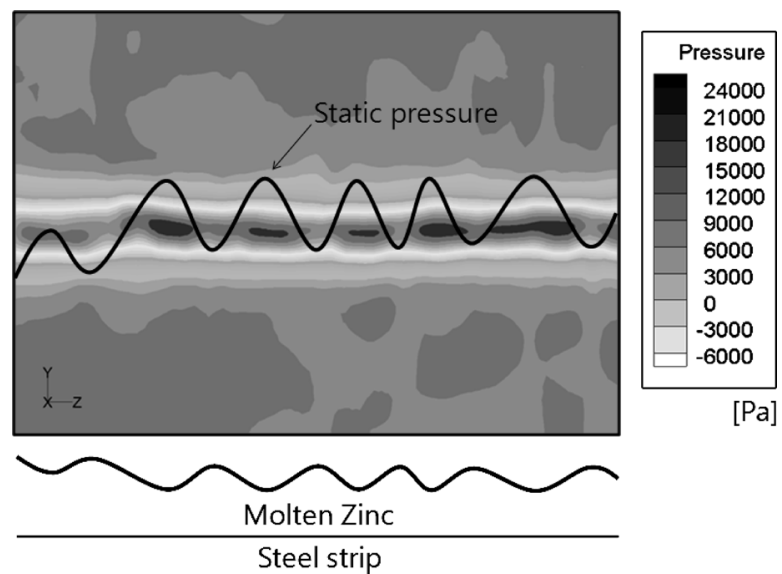

Fig. 9. Wave form of the molten zinc on the surface at the instantaneous time.

As a last picture of the flow field, Fig. 9 shows a detailed view of the variation of surface static pressure near the stagnation line. The black colors indicate the high pressure points. As can be seen, the high and low pressure points are appeared repeatedly. It is evident that more molten zinc is removed at the high pressure point in comparison with the low pressure point. $^{26)}$ Accordingly, the zinc coated steel strip would have a wavy surface as sketched in Fig. 9 at that instantaneous time.

\section{The Prediction of the Angle of Check-mark}

The sloping angle of the 3rd grade check-mark can be predicted by using the feeding speed of the steel strip and the sidewise velocity of the high pressure point on the stagnation line as shown in Fig. 10. The angle can be predicted by Eq. (2) that follows.

$$
\tan \theta=\frac{V_{\text {srip }}}{V_{p}}
$$

The sidewise velocity of the high pressure point was calculated by using the distance between the high pressure points and the period of high pressure appearance at the specific point on the stagnation line. The maximum value of the mean static pressure on the surface is about $18 \mathrm{kPa}$ (gauge) at $y=0.75 \mathrm{~mm}$. At this time, $y=0$ is taken at the lower end of the exit of the air knife. Since the exit width of the air knife is $1.5 \mathrm{~mm}$, therefore, this point at $y=0.75 \mathrm{~mm}$ is in the center of the jet. Subsequently, static pressure variation with time was used to calculate the sidewise velocity of the high pressure point. Figure 11(a) shows the variation of the static pressure at the middle point in the $z$-direction $(z=100 \mathrm{~mm})$ during $0.015 \mathrm{~s}$ and Fig. 11(b) demonstrates the instantaneous distribution of the instantaneous static pressure along the stagnation line at $y=0.75 \mathrm{~mm}$. The mean period of time between the appearance of the high pressure points is about $0.003 \mathrm{~s}$ from the Fig. 11(a) and the mean distance between the high pressure points is around $3 \mathrm{~mm}$ from the Fig. 11(b). Therefore, the sidewise velocity of the high pressure points is about $1 \mathrm{~m} / \mathrm{s}$ from the above results. The sloping angle of 70 degrees of the check-mark is obtained by substituting the above velocity and the moving velocity of the steel strip into Eq. (2). In Case 1, the sloping

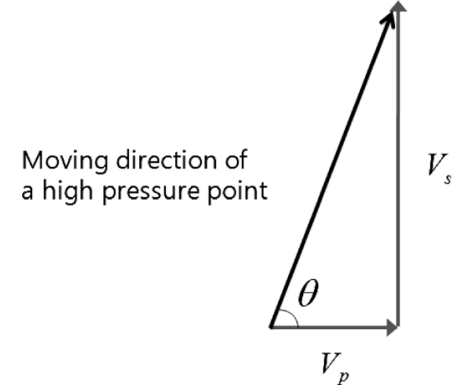

Fig. 10. Prediction of a angle of a check-mark.

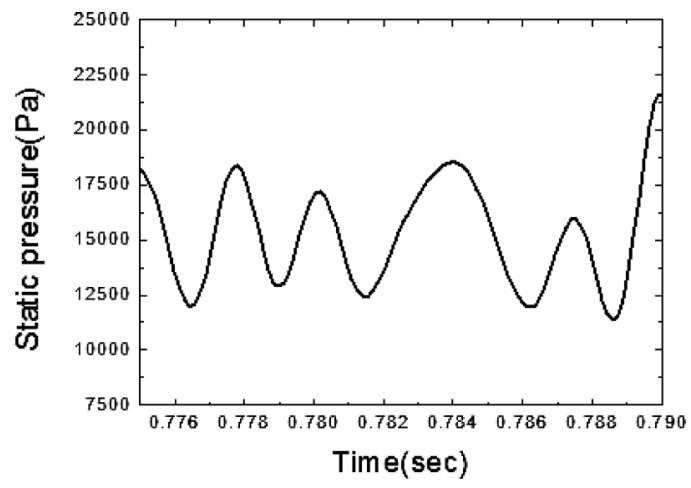

(a) Oscillation of static pressure on the surface at $y=0.75 \mathrm{~mm}, z=100 \mathrm{~mm}$

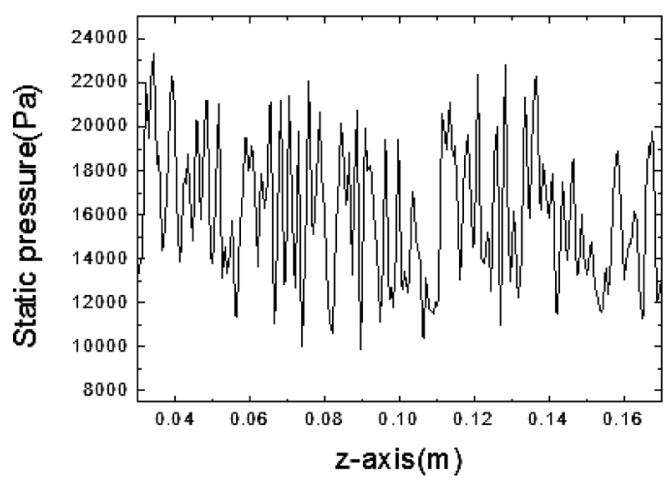

(b) Static pressure distribution along the $z$-direction at $\mathrm{y}=0.75$

Fig. 11. Oscillation of the static pressure with time and static pressure distribution along the stagnation line. (a) Oscillation of static pressure on the surface at $y=0.75 \mathrm{~mm}$, $z=100 \mathrm{~m}$. (b) Static pressure distribution along the $z$-direction at $y=0.75$.

angle and mean distance of the check-mark is about $75^{\circ}$ and $3 \mathrm{~mm}$ from the measured data, respectively. The numerical results are consistent with the measured data of the real check-mark stain within the experimental error range of about $10 \%$. By this favorable agreement between the measurement of the actual check-mark and the numerical prediction, the present theory about the cause of the check-mark stain is aptly justified.

Concerning the 2nd grade check-mark stain, although the check-mark pattern is a little different from the 3rd grade check-mark stain, the mechanism to cause the 2 nd grade stain is the same as that explained above for the 3rd grade stain. In the present study, Case 2 indicates the weak 2nd grade check-mark stain which has irregular patterns on the coating surface. The difference between the Case 1 and Case 2 comes from the ratio of $L$ to $d(L / d)$. The $L / d$ is 6.67 


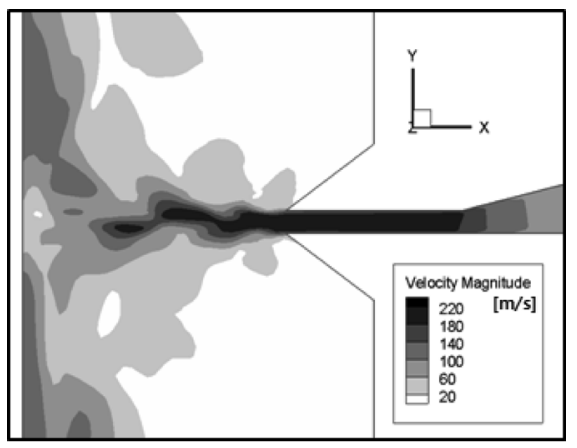

(a) Velocity magnitude contours

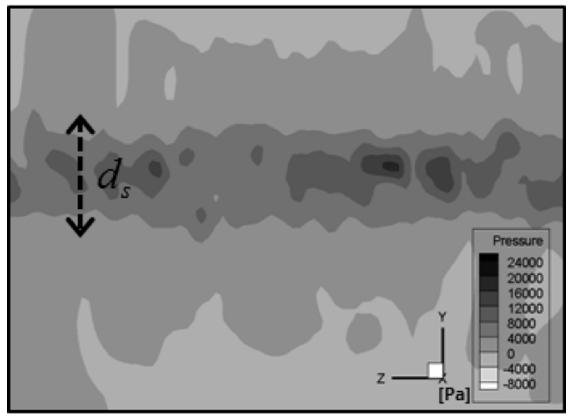

(b) Static pressure contours

Fig. 12. Velocity magnitude contours on the $x y$-plane and static pressure contours on the plate in the case 2. (a) Velocity magnitude contours, (b) static pressure contours.

in the Case 1 , but $L / d$ is 11.54 in the Case 2. Figure 12(a) displays the velocity field of the nitrogen gas on the $x y$ plane at the middle of the $z$-direction and Fig. 12(b) shows the static pressure contours on the $y z$-plane at $x=0.1 \mathrm{~mm}$. Contrary to the Case 1 as shown in Fig. 5, the transient region gets lengthen and the buckling of the jet is weak in the Case 2 as can be seen in Fig 12(a). Moreover the area of the impinging region is expanded due to the wider jet spreading. ${ }^{27,28)}$ If one defines an impingement pressure region on the strip surface is defined as the region from the stagnation line to the $y$-point where the surface static pressure becomes equal to the atmospheric pressure. The width of the impingement pressure region is about $10 \mathrm{~mm}$ in the Case 1. But, in the Case 2, its width is about $25 \mathrm{~mm}$ which is more than twice wider than that in the Case 1 . As the impinging region is expanded like this way, the repeated static pressure peaks and vortical structure no longer appears along the stagnation line, but the intermittent high pressure points appear as shown in Fig. 12(b). Consequently, much more irregular check-mark stain would be formed on the surface of the steel strip.

\section{The Schematic Picture of the Check-mark Stain}

The thickness of the coated zinc is approximately in inverse proportion to the impinging static pressure on the stagnation line. Therefore, the check-mark stain can be schematically drawn by tracing the changing locations of the locally peak pressures. In order to draw such lines of check-mark stain, static pressure data varying in time were used along the stagnation line at $y=0.75 \mathrm{~mm}$. The wavy thickness of the molten zinc formed on the surface at the

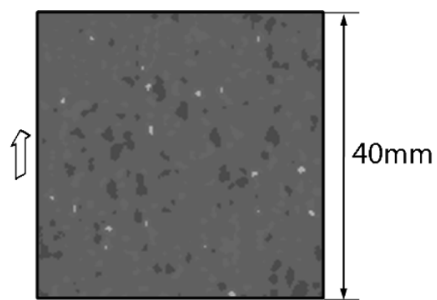

(a) Weak $2^{\text {nd }}$ grade check mark
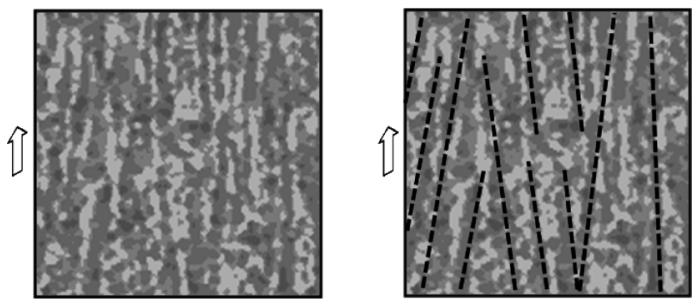

(b) $3^{\text {rd }}$ grade check mark

Fig. 13. The schematic picture of the check-mark by using the calculation results. (a) Weak 2nd grade check mark, (b) 3rd grade check mark.

instantaneous time is moved upward with the same velocity of the steel strip. In this way, the thinnest locations can be traced in $y z$ plane. The distance that a particular point travels in $y$-direction is obtained by the following Eq. (3)

$$
D_{v}=\Delta t \times V_{\text {strip }} \times N
$$

Where $\Delta t$ is the computational time step, $V_{\text {strip }}$ is the upward moving speed of the steel strip and $N$ is the number of computation steps.

The schematic pictures of the check-mark stain obtained by using the above method are displayed in Fig. 13. Each arrow indicates the moving direction of steel strip. The dark gray indicates the area of thin coated thickness and bright gray indicates the area of thick coated thickness. Figure 13(a) represents the weak 2 nd grade check-mark stain and Fig. 13(b) represents the 3rd grade check-mark stain. The schematic picture of the check-mark stain in Fig. 13(b) resembles the actual 3rd grade check-mark stain.

\section{Conclusion}

In order to investigate the cause of the check-mark stain problem in a continuous hot-dip galvanizing process, the 3 $\mathrm{D}$ flow field around the air knife and the moving steel strip is calculated by using the commercial code, FLUENT. LES technique was used to simulate as exactly as possible the unstable and complex 3-D flow flied established by impinging a two-dimensional plane jet normally onto a moving steel strip in a relatively short distance. As a result of our simulation, it was observed that high pressure and low pressure appear almost cyclically along the impingement stagnation line. The slope of the check-mark stain could be predicted relatively accurately by using the feeding speed of the steel strip and the sidewise velocity of the high pressure points along the stagnation line. The computed distance and slope of the check-mark stain were remarkably consistent with experimental measurements. The surface of the molten zinc has the wavy form because more molted zinc is re- 
moved at the point of high static pressure in comparison with that at the low pressure points. It is shown that the schematic picture of the check-mark stain depicted by using the numerical data about the variation of surface static pressure in time and space and the feeding speed of the steel strip resembles the pattern of the actual check-mark stain. Such a favorable comparison between the predicted pattern of the check-mark stain and the actual stain aptly justifies the present theory about the cause of the check-mark stain. In the near future, this theory may be usefully applied to find an engineering technique to completely resolve the check-mark stain problem in the hot dip galvanizing process.

\section{Acknowledgement}

The authors acknowledge the financial support from POSCO for the present study in 2007.

\section{Nomenclature}

$D_{v}$ : Vertical length of the schematic picture of the check-mark (mm)

$F_{r}:$ Reaction force on the jet

$F_{c}$ : Compressive force on the jet

$N$ : Number of computation steps

$V_{p}$ : Sidewise moving velocity of a high pressure point $(\mathrm{m} / \mathrm{s})$

$v_{\text {strip }}$ : Moving velocity of the steel strip $(2.5 \mathrm{~m} / \mathrm{s})$

$L:$ Distance from the nozzle exit (lip of the air knife) to the steel strip (mm)

$d$ : Lip width of the air knife (mm)

$d_{s}$ : Width of the stagnation line

$\lambda_{B}$ : Buckling wavelength of the jet

$\theta$ : Angle of an oblique line

\section{REFERENCES}

1) J. M. Buchlin, M. Manna, M. Arnalsteen, M. L. Riethmuller and M. Dubois: European Coating Symp. on the Mechanism of Thin Film Coating, World Scientific, Singapore, (1996), 168.

2) J. A. Thornton and H. F. Graff: Metall. Mater. Trans. B, 7B (1976),
607.

3) C. H. Ellenand and C. V. Tu: J. Fluids Eng., 106 (1984), 399.

4) Y. Takeishi, A. Yamauchi and S. Miyauchi: Tetsu-to-Hagané, 80 (1995), 37.

5) J. R. Park, S. H. Jeon and R. B. Park: J. Korea Institute of Surface Eng., 32 (1999), 555.

6) Y. Takeishi and H. Morino: ISIJ Int., 40 (2000), 1127.

7) S. J. Kim, J. W. Cho, G. J. Ahn and M. K. Chung: ISIJ Int., 43 (2003), No. 10, 1495.

8) G. J. Ahn and M. K. Chung: ISIJ Int., 46 (2006), No. 4, 573.

9) B. M. Tchavdarov: Int. J. Heat Fluid Flow, 18 (1996), 316.

10) Y. M. Chung, K. H. Luo and N. D. Sandham: Int. J. Heat Fluid Flow, 23 (2002), 592.

11) A. C. Victor and O. Alfonso: Int. J. Heat Mass Transfer, 45 (2002), 1237.

12) V. Narayanan, J. Seyed-Yagoobi and R. H. Page: Int. J. Heat Mass Transfer, 47 (2004), 1827.

13) V. Narayanan and V. A. Patil: Exp. Thermal Fluid Sci., 32 (2007), 682.

14) S. D. Hwang, C. H. Lee and H. H. Cho: Int. J. Heat Fluid Flow, 22 (2001), 293.

15) M. Tsubokura, T. Kobayashi, N. Taniguchi and W. P. Jones: Int. J. Heat Fluid Flow, 24 (2003), 500.

16) H. K. Versteeg and W. Malalasekera: Computational Fluid Dynamics, Pearson Prentice Hall, London, (2007), 98.

17) R. W. Fox and A. T. Mcdonald: Introduction to Fluid Mechanics, Wiley, New York, (1998), 604.

18) J. Senter and C. Solliec: Int. J. Heat Fluid Flow, 28 (2007), 708.

19) M. A. R. Sharif and A. Banerjee: Appl. Thermal Eng., 29 (2009), 532.

20) A. Bejan: Entropy Generation through Heat and Fluid Flow, John Wiley \& Sons, New York, (1982), 64.

21) T. Cziesla, G. Biswas, H. Chattopadhyay and N. K. Mitra: Int. J. Heat Fluid Flow, 22 (2001), 500.

22) G. L. Song and M. Prud'homme: Int. J. Heat Fluid Flow, 28 (2007), 1125.

23) X. Jiang, H. Zha and K. H. Luo: Computers Fluids, 36 (2007), 259.

24) M. H. Ching and N. S. Nosseir: J. Fluid Mech., 105 (1981), 119.

25) T. Naito, O. Banno and H. Yamada: Fluid Dynamics Res., 28 (2001), 61

26) A. Katsuya and O. Tsuyoshi: 7th International Conference on Zinc and Zinc Alloy Coated Steel Sheet, ISIJ, Tokyo, (2007), 177.

27) D. C. Wilcox: Turbulence Modeling for CFD, DCW Industries, Canada, (2007), 72.

28) F. Beaubert and S. Viazzo: Int. J. Heat Fluid flow, 24 (2003), 512. 